OPEN ACCESS

Edited by:

Xueming Liu,

Zhejiang University, China

Reviewed by:

Xiaohui Li,

Shaanxi Normal University, China

Zhi-Chao Luo,

South China Normal University, China

*Correspondence:

Chen Wei

cwei@uestc.edu.cn

Specialty section:

This article was submitted to

Optics and Photonics,

a section of the journal

Frontiers in Physics

Received: 29 September 2019

Accepted: 17 October 2019

Published: 01 November 2019

Citation:

Lyu Y, Wei C, Du W, Li H and Liu Y (2019) Splitting and Motions of Dissipative Soliton Resonance Pulses in Mode-Locked Fiber Lasers. Front. Phys. 7:173

doi: 10.3389/fphy.2019.00173

\section{Splitting and Motions of Dissipative Soliton Resonance Pulses in Mode-Locked Fiber Lasers}

\author{
Yanjia Lyu, Chen Wei*, Wenxiong Du, Heping Li and Yong Liu \\ State Key Laboratory of Electronic Thin Films and Integrated Devices, School of Optoelectronic Information, University of \\ Electronic Science and Technology of China, Chengdu, China
}

We numerically investigated the splitting and motions of dissipative soliton resonance (DSR) pulses in an all-normal-dispersion Yb-doped fiber laser mode-locked by a non-linear optical loop mirror. At certain values of the system parameters, the initial single Gaussian pulse can evolve into an unstable DSR pulse with several dark solitons inside. After collisions of dark solitons, a big intensity dip occurs at the center of the DSR pulse, leading to the splitting of the DSR pulse. After splitting, the peak-to-peak separation between two DSR pulses increases at first and finally reaches a fixed value of 193 ps, indicating a steady-state multipulse operation The relative motions of separated DSR pulse originate from the phase shift.

Keywords: fiber lasers, mode-locked lasers, dissipative soliton resonance, non-linear optics, pulse splitting

\section{INTRODUCTION}

Passively mode-locked fiber lasers have attracted considerable attention due to their compact and stable performance for generating ultrafast pulse with high peak power [1-4]. As powerful tools, they also open an interest among researchers to explore new areas of soliton build-up dynamics [510]. Recently, a novel pulse formation known as dissipative soliton resonance (DSR) was initially found in the frame of complex cubic-quantic Ginzburg-Landau equation (CQGLE) in a specific parameter space [11]. Relying on the delicate and complicated balance of dispersion, non-linearity, loss, gain, non-linear gain saturation, and gain dispersion, the pulses in the DSR regime exhibit flattop profiles and wave-breaking-free features. With increasing pump power, the DSR pulse keeps broadening its pulse width with a clamped peak power without suffering pulse breaking, which shows great potential for high-energy pulse generation in fiber lasers. During the past decade, the DSR phenomenon has been extensively investigated theoretically and experimentally with different dispersion regimes, mode-locking techniques and emission wavelengths [12-30]. It was found that the peak-power-clamping effect caused by reverse saturable absorption plays an important role in the formation of DSR pulses.

Although in the DSR regime light pulses are supposed to be wave-breaking-free, multipulse operation is still possible. In 2013, Komarov et al. have numerically demonstrated that an initial multipulse field with different amplitudes can evolve into a steady-state multipulse operation in DSR regime, where the number of pulses depends on the initial conditions [31]. In 2016, Armas-Rivera et al. have observed that a single DSR pulse splits into two equidistant trapezoidalshaped DSR pulses by roundtrip at sufficiently high pump power [32]. In 2017, we have developed a harmonic DSR Er-dope fiber laser mode-locked by non-linear polarization rotation and numerically investigated its multipulse dynamics $[33,34]$. Recently, Chowdhury et al. have observed DSR pulse splitting by rotating polarization controllers in an all-normal dispersion 
non-linear amplifying loop mirror based mode-locked Yb-doped fiber laser, and they have also numerically studied the multipulse dynamics of DSR with multipulse input field [35]. Very recently, Wang et al. have observed unusual evolutions of DSR in an allnormal dispersion fiber laser, where increasing pump power leads to pulse breaking and multipulse operation [36]. However, the entire splitting process from one single pulse to multiple DSR pulses and its mechanism has not been numerically demonstrated so far.

In this paper, we numerically investigated the DSR pulse splitting process in a non-linear optical loop mirror (NOLM) mode-locked Yb-doped fiber laser using the scalar non-linear Ginzburg-Landau equation. It is found that, with specific parameter selection, the initial single pulse can evolve into an unstable DSR pulse with several dark solitons inside, and then split into two stable DSR pulses with the same pulse properties (pulse shape, duration, peak power, chirp, and so on) and fixed peak-to-peak (PP) separation. This steady-state two DSR pulses operation can be attributed to energy quantization effect [37] and phase shift of pulses [38].

\section{NUMERICAL MODEL}

The schematic of the proposed NOLM mode-locked Yb-doped fiber laser is shown in Figure 1. The cavity consists of a 1-mlong $\mathrm{Yb}$-doped fiber (YDF), 5-m-long single mode fiber (SMF), a saturable absorber (SA) with a sinusoidal transmission function, and a 90:10 optical coupler (OC) with $10 \%$ output. To take every component in the cavity into account, our simulations are still based on the pulse tracing technique. The simulations start from a weak single Gaussian pulse. The pulse propagation in the fiber sections is modeled by a scalar non-linear GinzburgLandau equation:

$$
\frac{\partial A}{\partial z}=-\frac{i \beta_{2}}{2} \frac{\partial^{2} A}{\partial t^{2}}+i \gamma|A|^{2} A+\frac{g}{2} A+\frac{g}{2 \Omega_{g}^{2}} \frac{\partial^{2} A}{\partial t^{2}}
$$

where $A$ is the slowly varying electric field envelope, $z$ is the propagation distance, and $t$ is the pulse local time. $\beta_{2}$ and $\gamma$ refer to the group velocity dispersion and the non-linearity of fiber, respectively. In the simulation all the fibers are assumed to have the same non-linearity of $\gamma=3 \mathrm{~W}^{-1} \cdot \mathrm{km}^{-1}$, while the dispersion parameters are $\beta_{2, Y D F}=20 \mathrm{ps}^{2} \cdot \mathrm{km}^{-1}, \beta_{2, S M F}=22 \mathrm{ps}^{2} \cdot \mathrm{km}^{-1}$, For the YDF, $g$ is the saturable gain of the fiber and $\Omega_{g}=40 \mathrm{~nm}$ is the gain bandwidth with a parabolic shape. For the SMF, $g=0$. For

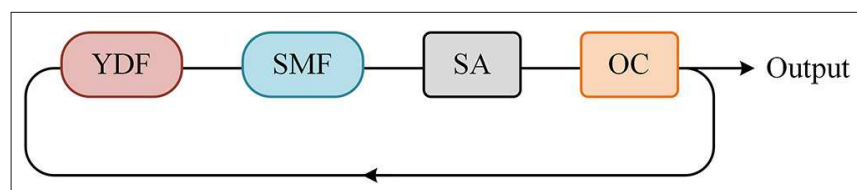

FIGURE 1 | Schematic of the NOLM mode-locked Yb-doped fiber laser. the YDF, the gain saturation effect is considered as

$$
g=g_{0} \exp \left(-\frac{1}{E_{\text {sat }}} \int\left|A^{2}\right| d t\right)
$$

where $g_{0}=3 \mathrm{~m}^{-1}$ is the small signal gain coefficient, which relates to the doping concentration of the gain fiber; $E_{\text {sat }}$ refers to the gain saturation energy, which relates to the pumping strength.

In our simulations, a simple model of NOLM is adopted, and its transmission function can be expressed as:

$$
T=\frac{1}{2}\left[1-q \cos \left(\frac{\pi\left(1-\Phi_{0}\right)|A|^{2}}{I_{\text {sat }}}+\Phi_{0} \pi\right)\right]
$$

where $q$ is the modulation depth, $\Phi_{0}$ accounts for linear bias, and $I_{\text {sat }}$ is the saturation power. In our simulations, we set $q=0.4, \Phi_{0}$ $=0$, and $I_{\text {sat }}=40 \mathrm{~W}$.

\section{SIMULATION RESULTS AND DISCUSSION}

Several previous theoretical works have studied the multipulse dynamics of DSR [31, 33, 35]. To generate multiple DSR pulses, all these simulations required a multipulse initial field, and during the evolution from the initial field to multiple DSR pulses, no pulse splitting process was found. To investigate the pulse splitting process of DSR, in our simulations we use only one single Gaussian pulse with weak peak power as the initial field.

The pulse splitting process of DSR was observed when the pump strength was selected as $E_{s a t}=2 \mathrm{~nJ}$. The $2 \mathrm{D}$ Color fill and $3 \mathrm{D}$ color map surface of the pulse evolution are shown in Figures 2A,B, respectively. From Figure 2A, we can obviously see that after a chaos process, one pulse splits into two stable DSR pulses. The PP separation between these two DSR pulses increases rapidly at the beginning of roundtrips, and eventually, it reaches a value of $\sim 193 \mathrm{ps}$ and keeps constant after the roundtrip number $N>4,000$. Figure $2 \mathrm{C}$ shows the temporal profiles of the two DSR pulse at $N=5,000$. The two DSR pulse have the same shape, pulse duration of $\sim 41 \mathrm{ps}$, and peak power of $\sim 5.5 \mathrm{~W}$. As shown in Figure 2D, the two DSR pulses still have the same instantaneous frequency profiles. The pulses have non-zero linear frequency chirps across their flat-top part and exponential chirps at the edges, which are essential features of DSR.

For better understanding the pulse splitting phenomenon in DSR regime, we focused on the evolutions of the first 500 roundtrips. Figures $\mathbf{3 A}$, B show the pulse evolution of the first 500 roundtrips when $E_{\text {sat }}$ was set to $2 \mathrm{~nJ}$ and $1.98 \mathrm{~nJ}$, respectively. When $E_{\text {sat }}=2 \mathrm{~nJ}$, after a chaos process the initial input pulse firstly evolves into an unstable DSR pulse with several dark solitons inside it. These dark solitons evolved from noise perturbations are commonly observed in dissipative soliton fiber lasers, since they are the stable solutions of the nonlinear Schrodinger equation in the normal dispersion regime. When dark solitons are formed inside a dissipative soliton, the sloped background intensity will impose additional phase shifts on them, resulting in varying transverse velocities, which is proportional to the background intensity. Consequently, dark solitons in a dissipative soliton always walk off from the bright 

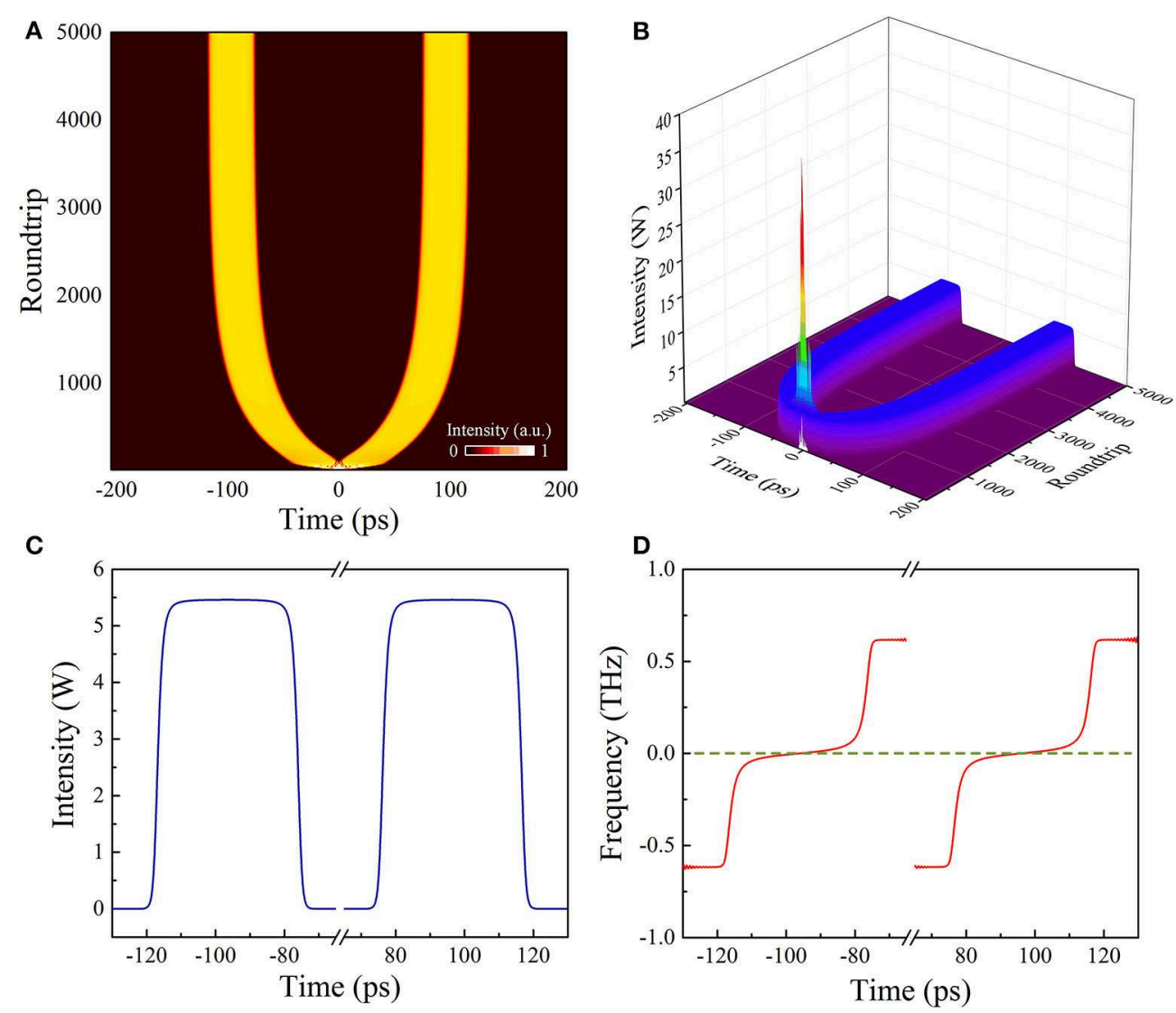

FIGURE 2 | Pulse splitting process of DSR at $E_{\text {sat }}=2$ nJ. (A) 2D Color fill, (B) 3D color map surface of evolutions. (C) Temporal profiles and (D) instantaneous frequency profiles at $N=5000$.
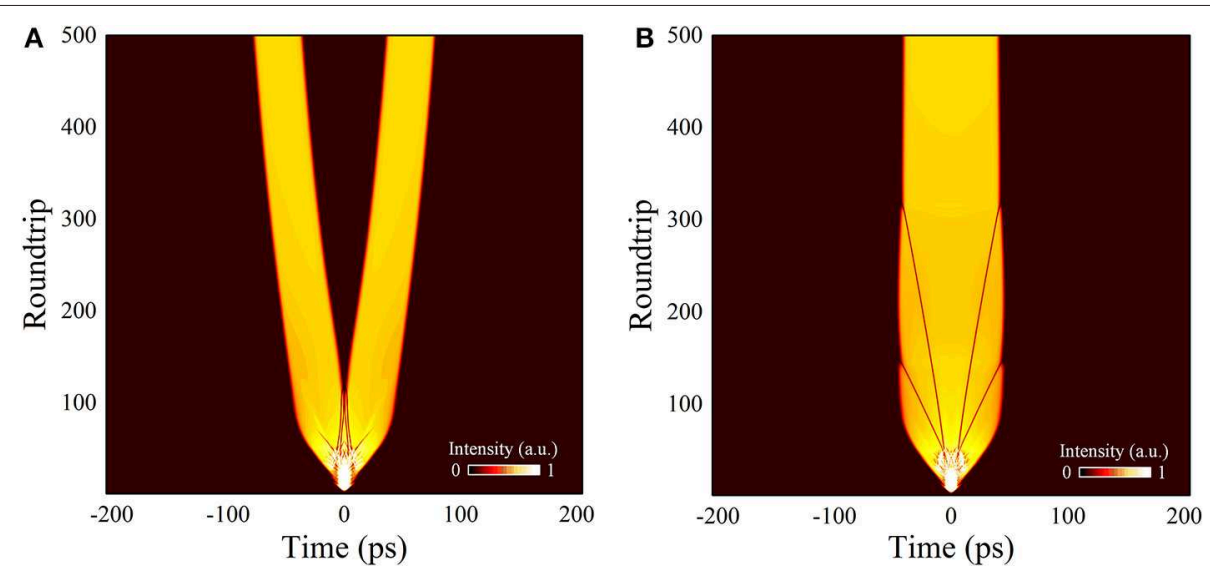

FIGURE 3 | Pulse evolution of first 500 roundtrips at (A) $E_{\text {sat }}=2 \mathrm{~nJ}$, (B) $E_{\text {sat }}=1.98 \mathrm{~nJ}$.

pulse and eventually decay with curved trajectories toward wings of the bright pulse [39]. However, in the DSR square-shaped pulse in our simulation, the formed dark solitons are on a quasi$\mathrm{cw}$ background with almost constant intensity level, and thus no additional phase shift is imposed on them, making them move with constant transverse velocities (in other words, they have straight trajectories). This make the dark solitons possible to avoid decaying at the wings of the background pulse. Here, as shown in Figure 3A, although 2 gray solitons decay in the background, 4 dark solitons move straight toward the center of the background DSR pulse, and after collisions they finally converge into one big intensity dip when the roundtrip number 

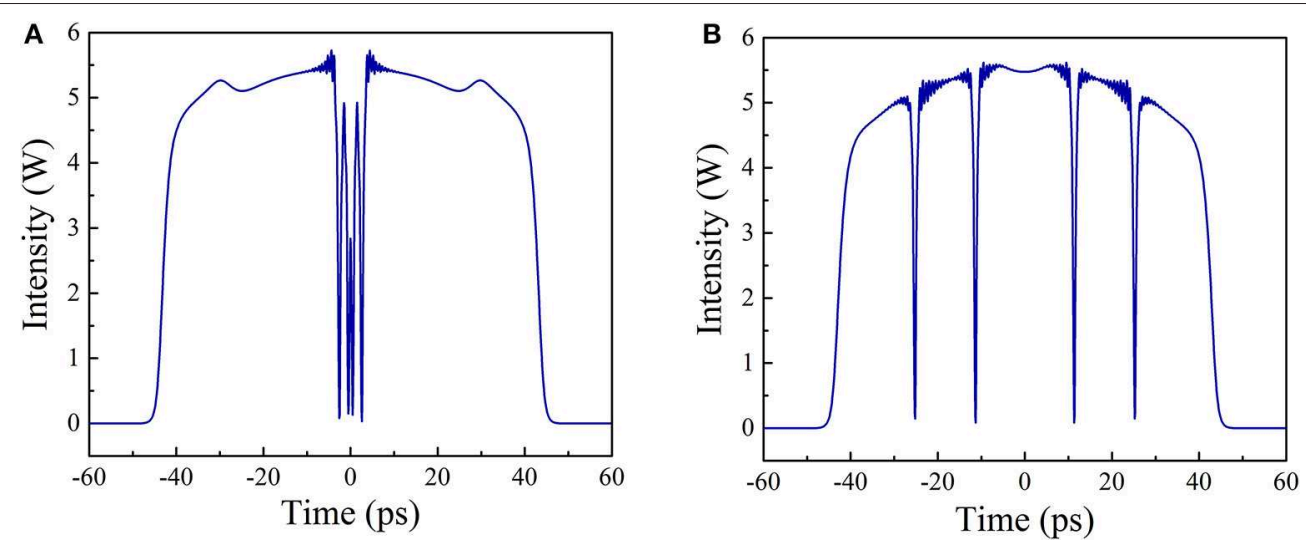

FIGURE $4 \mid$ Pulse temporal profiles at $N=100$ at $\mathbf{( A )} E_{\text {sat }}=2 \mathrm{~nJ}$, (B) $E_{\text {sat }}=1.98 \mathrm{~nJ}$.
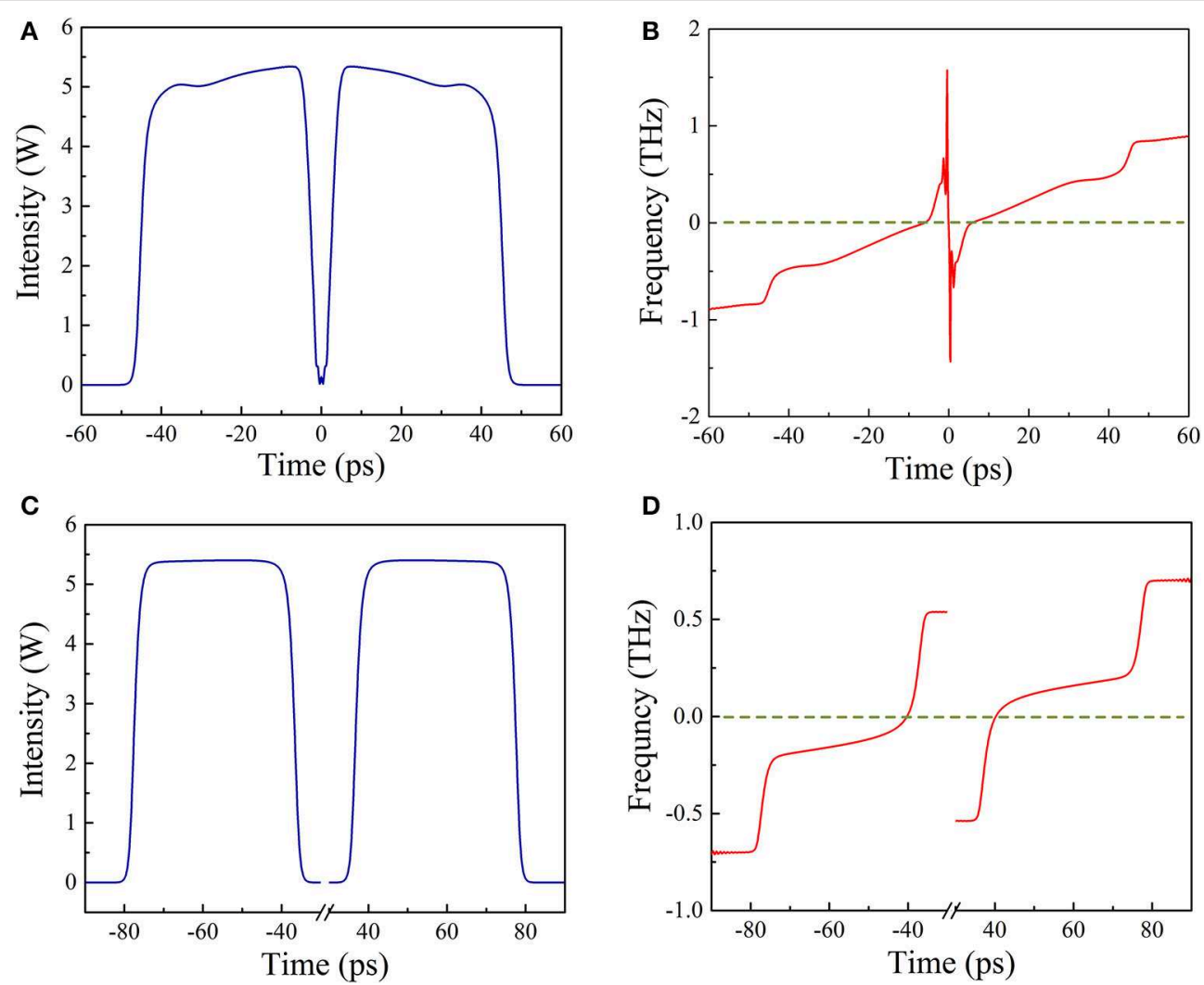

FIGURE 5 | Pulse properties at different roundtrips when $E_{\text {sat }}=2 \mathrm{~nJ}$. (A) Temporal profile at $N=120$. (B) Instantaneous frequency profile at $N=120$. (C) Temporal profile at $N=500$. (D) Instantaneous frequency profile at $N=500$.

$N=115$. As a consequence, the left and right parts of the unstable broken DSR pulse are separated by this intensity dip, and these two parts become individual DSR pulses with the same properties. Since the dark solitons in the system originate from random spontaneous noise in the early build-up process of the DSR pulse, they would emerge from the background with varying position and blackness, which make it difficult to control the system parameters in our simulations to realize DSR pulse splitting. When we set system parameters (e.g., $E_{\text {sat }}, \beta_{2}$, and $\gamma$ ) to many other different values, even very close to the original values, the initial pulse always evolves into one stable single DSR pulse, in other words, the dark solitons decay at wings of the DSR pulse. As shown in Figure 3B, when $E_{\text {sat }}=1.98 \mathrm{~nJ}$, the initial input pulse can still evolve into an DSR pulse with 4 dark solitons. However, in this case, all the dark solitons move straight toward the DSR pulse wings and eventually decay, leading to the 


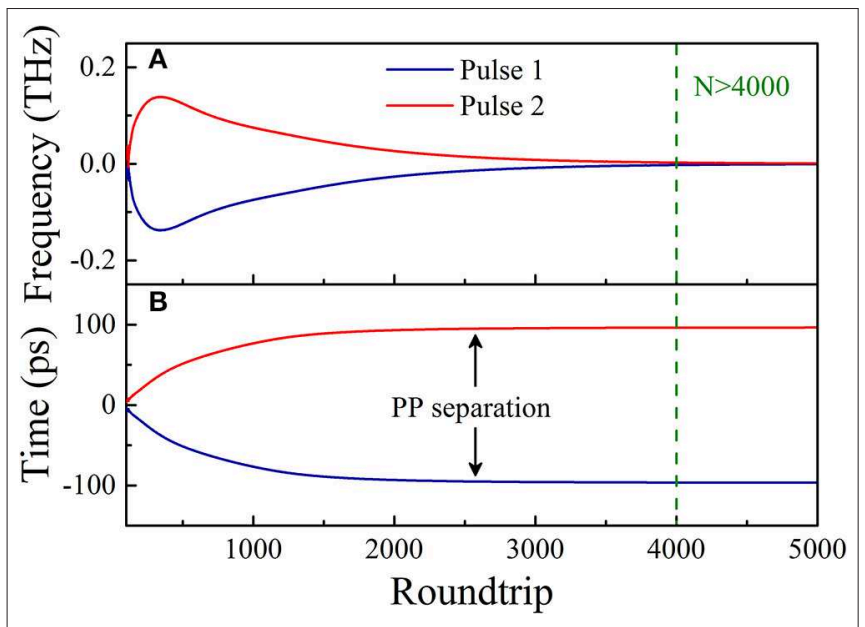

FIGURE 6 | (A) Instantaneous frequency at pulse peak $F_{P}$ and (B) pulse trajectory vs. roundtrip number $N$.

formation of one stable DSR pulse. The results indicate that the DSR pulse splitting depends strongly on the system parameters, and the physical mechanism requires further investigation.

Figure $4 \mathrm{~A}$ shows the pulse temporal profile at $N=100$ and $E_{\text {sat }}=2 \mathrm{~nJ}$. Here we observe the gathering of the 4 dark solitons at the center of the DSR pulse. As the collisions of dark solitons decrease the background intensity, their blackness also decrease. It is not surprising that they would eventually decay and form a big gap in the DSR pulse, leading to the splitting process. While for $N=100$ and $E_{\text {sat }}=1.98 \mathrm{~nJ}$, as shown in Figure 4B, the dark solitons are separated and have no chance of collisions. It should be noted that the splitting mechanism of DSR pulse here is entirely different from that of conventional soliton. Increasing pump strength cannot lead to the splitting of the DSR pulse but only leads to the broadening of the pulse width.

The relative motions of the two DSR pulses are similar to that of solitons in Liu [38], which can also be attributed to the phase shift arising from pulse interactions. When the phase shift is imposed on a pulse, the instantaneous frequency at the pulse peak, $F_{P}$, becomes non-zero, as the instantaneous frequency is the first derivative of phase. Figure $\mathbf{5 A}$ shows the temporal profile at $N=120$ when $E_{\text {sat }}=2 \mathrm{~nJ}$, where the broken DSR pulse is just separated into two symmetrical trapezoid-shaped DSR pulses. Figure 5B shows the corresponding instantaneous frequency at $N=120$. Obviously, both pulses have almost linear frequency chirps with relatively large slopes across their pulse profiles. For the left trapezoid-shaped DSR pulse, its $F_{P}<0$, and for the right one its $F_{P}>0$, indicating that both pulses have phase shifts. After hundreds of roundtrips, as shown in Figure 5C, at $N=500$ the trapezoid-shaped pulses transform to the typical square-shaped DSR pulses and they separate from each other.

\section{REFERENCES}

1. Zhao Y, Guo P, Li X, Jin Z. Ultrafast photonics application of graphdiyne in optical communication region. Carbon. (2019) 149:336-41. doi: 10.1016/j.carbon.2019.04.075
Figure 5D shows the corresponding instantaneous frequency at $N=500$. Each pulse still has linear frequency chirp across the pulse profile, but the slope decrease and the $F_{P}$ becomes closer to zero as compared with trapezoid-shaped DSR pulse at $N=120$. As shown in Figures 2C,D, at $N=5,000$, these two DSR pulses reach a steady-state multipulse operation with a fix $\mathrm{PP}$ separation of $\sim 193$ ps and the $F_{P}$ of each pulse is almost equals to zero.

Figures 6A,B show the evolutions of the $F_{P}$ and pulse trajectory from $N=120$ to $N=5,000$. The absolute value of each $F_{P}$ increases at the beginning, and then it keeps decreasing and eventually reaches zero at $N=4,000$. Meanwhile, the PP separation between two pulses keeps increasing and gradually approaches $\sim 193$ ps at $N=4,000$. When $N>4,000$, the $F_{P}$ is fixed at 0 and $\mathrm{PP}$ separation is fixed at $\sim 193 \mathrm{ps}$, indicating a steadystate multipulse operation. Obviously, the motions of two pulses are governed by the $F_{P}$ or the phase shift, in other words.

\section{CONCLUSION}

In conclusion, we have numerically studied the pulse splitting process and their motions in DSR regime in an Yb-doped fiber laser mode-locked by NOLM. Numerical simulations have demonstrated that with specific system parameters selection, one unstable DSR pulse with several dark solitons inside can split into two DSR pulse with the same properties due to the collisions and decay of dark solitons. The pulses can eventually reach a steadystate operation with a fixed separation. Similar to conventional solitons, the phase shift governs the relative motions of the separated DSR pulses. We believe that our simulation results can offer an insight into DSR pulse dynamics in mode-locked fiber lasers

\section{DATA AVAILABILITY STATEMENT}

The data used to support the findings of this study are available from the corresponding author upon request.

\section{AUTHOR CONTRIBUTIONS}

YLy contributed conception and design of the study. CW helped to evaluate and edit the manuscript. CW and YLi supervised the project. All the authors contributed to discussion on the results for this manuscript.

\section{FUNDING}

This work was supported by the National Nature Science Foundation of China (NSFC) (61435003, 61421002, 61875033, 61775031), Chengdu Science and Technology Huimin Project (2016-HM01-00269-SF), and China Scholarship Council (201706070044).
2. Liu J, Li X, Guo Y, Qyyum A, Shi Z, Feng T, et al. SnSe 2 Nanosheets for subpicosecond harmonic mode-locked pulse generation. Small. (2019) 1902811:1-7. doi: 10.1002/smll.201902811

3. Guo P, Li X, Chai T, Feng T, Ge Y, Song Y, et al. Few=layer bismuthene for robust ultrafast photonics in C-Band optical 
communications. Nanotechnology. (2019) 30:354002. doi: 10.1088/1361-6528/ ab2150

4. Feng J, Li X, Qyyum A, Zhang Y, Zheng C, Wang Y, et al. SnS nanosheets for 105th harmonic soliton molecule generation. Ann Phys. (2019) 531:1900273. doi: 10.1002/andp.201900273

5. Wei ZW, Liu M, Ming SX, Luo AP, Xu WC, Luo ZC. Pulsating soliton with chaotic behavior in a fiber laser. Opt Lett. (2018) 43:5965-8. doi: 10.1364/OL.43.005965

6. Yu Y, Luo ZC, Kang JQ, Wong KY, Mutually ignited soliton explosions in a fiber laser. Opt Lett. (2018) 43:4132-5. doi: 10.1364/OL.43.004132

7. Liu X, Yao X, Cui Y. Real-time observation of the buildup of soliton molecules. Phys Rev Lett. (2018) 121:023905. doi: 10.1103/PhysRevLett.121.023905

8. Liu X, Popa D, Akhmediev N. Revealling the transition dynamics from Q switching to mode locking in a soliton laser. Phys Rev Lett. (2019) 123:093901. doi: 10.1103/PhysRevLett.123.093901

9. Liu X, Cui Y. Revealing the behavior of soliton buildup in a mode-locked laser. Adv Photonics. (2019) 1:016003. doi: 10.1117/1.AP.1.1.016003

10. Liu X, Pang M. Revealing the buildup dynamics of harmonic modelocking states in ultrafast lasers. Laser Photonic Rev. (2019) 13:1800333. doi: 10.1002/lpor.201800333

11. Chang W, Ankiewicz A, Soto-Crespo JM, Akhmediev N. Dissipative soliton resonances. Phys Rev A. (2008) 78:023830. doi: 10.1103/PhysRevA.78.023830

12. Chang W, Soto-Crespo JM, Ankiewicz A, Akhmediev N. Dissipative soliton resonances in the anomalous dispersion regime. Phys Rev A. (2009) 79:033840. doi: 10.1103/PhysRevA.79.033840

13. Liu $X$. Pulse evolution without wave breaking in a strongly dissipative-dispersive laser system. Phys Rev A. (2010) 81:053819. doi: 10.1103/PhysRevA.81.053819

14. Grelu P, Chang W, Ankiewicz A, Soto-Crespo JM, Akhmediev N. Dissipative soliton resonance as a guideline for high-energy pulse laser oscillators. J Opt Soc Am B. (2010) 27:2336-41. doi: 10.1364/JOSAB.27.002336

15. Ding E, Grelu P, Kutz JN. Dissipative soliton resonance in a passively modelocked fiber laser. Opt Lett. (2011) 36:1146-48. doi: 10.1364/OL.36.001146

16. Li D, Tang D, Zhao L, Shen D. Mechanism of dissipative-soliton-resonance generation in passively mode-locked all-normal-dispersion fiber lasers. J Light Technol. (2015) 33:3781-87. doi: 10.1109/JLT.2015.2449874

17. Liu X. Coexistence of strong and weak pulses in a fiber laser with largely anomalous dispersion. Opt Express. (2011) 19:5874-87. doi: 10.1364/OE.19.005874

18. Luo ZC, Cao WJ, Lin ZB, Cai ZR, Luo AP, Xu WC. Pulse dynamics of dissipative soliton resonance with large duration-tuning range in a fiber ring laser. Opt Lett. (2012) 37:4777-9. doi: 10.1364/OL.37.004777

19. Wu X, Tang DY, Zhang H, Zhao LM. Dissipative soliton resonance in an allnormal-dispersion erbium-doped fiber laser. Opt Express. (2009) 17:5580-4. doi: 10.1364/OE.17.005580

20. Duan L, Liu X, Mao D, Wang L, Wang G. Experimental observation of dissipative soliton resonance in an anomalous-dispersion fiber laser. Opt Express. (2012) 20:265-70. doi: 10.1364/OE.20.000265

21. Mei L, Chen G, Xu L, Zhang X, Gu C, Sun B, et al. Width and amplitude tunable square-wave pulse in dual-pump passively mode-locked fiber laser. Opt Lett. (2014) 39:3235-7. doi: 10.1364/OL.39.003235

22. Wang SK, Ning QY, Luo AP, Lin ZB, Luo ZC, Xu WC. Dissipative soliton resonance in a passively mode-locked figure-eight fiber laser. Opt Express. (2013) 21:2402-7. doi: 10.1364/OE.21.002402

23. Zhao N, Liu M, Liu H, Zheng XW, Ning QY, Luo AP, et al. Dualwavelength rectangular pulse Yb-doped fiber laser using a microfiberbased graphene saturable absorber. Opt Express. (2014) 22:10906-13. doi: 10.1364/OE.22.010906

24. Guo B, Yao Y, Yang YF, Juan YJ, Jin L, Yan B, et al. Dual-wavelength rectangular pulse erbium-doped fiber laser based on topological insulator saturable absorber Photon Res. (2015) 3:94-9. doi: 10.1364/PRJ.3.0 00094

25. Krzempek K. Dissipative soliton resonance in all-fiber Er:Yb double clad figure-8 laser. Opt Express. (2015) 23:30651-6. doi: 10.1364/OE.23.030651

26. Semaan G, Braham FB, Salhi M, Meng Y, Bahloul F, Sanchez F. Generation of high energy square-wave pulses in all anomalous dispersion Er: $\mathrm{Yb}$ passive mode locked fiber ring laser. Opt Express. (2016) 24:8399-404. doi: 10.1364/OE.24.008399

27. Krzempek K, Abramski K. Dissipative soliton resonance mode-locked double clad Er:Yb laser at different values of anomalous dispersion. Opt Express. (2016) 24: 22379-86. doi: 10.1364/OE.24.022379

28. Du T, Luo Z, Yang R, Huang Y, Ruan Q, Cai Z, et al. 1.2-W averagepower, 700-W peak-power, 100-ps dissipative soliton resonance in a compact Er:Yb co-doped double-clad fiber laser. Opt Lett. (2017) 42:462-5. doi: 10.1364/OL.42.000462

29. Du W, Li H, Li J, Wang P, Zhang S, Liu Y. Mechanism of dissipative-solitonresonance generation in fiber laser mode-locked by real saturable absorbe. Opt Express. (2018) 26:21314-23. doi: 10.1364/OE.26.021314

30. Du W, Li H, Li J, Wang Z, Wang P, Zhang Z, et al. Polarzation dynamics of dissiative-solition-resonance pulses in passively mode-locked fiber lasers. Opt Express. (2019) 27:8059-68. doi: 10.1364/OE.27.008059

31. Komarov A, Amrani F, Dmitriev A, Komarov K, Sanchez F. Competition and coexistence of ultrashort pulses in passive mode-locked lasers under dissipative-soliton-resonance conditions. Phys Rev. A. (2013) 87:023838. doi: 10.1103/PhysRevA.87.023838

32. Armas-Rivera I, Cuadrado-Laborde C, Carrascosa A, Kuzin EA, Beltran-Perez G, Diez A. Dissipative soliton resonance in a full polarization-maintaining fiber ring laser at different values of dispersion. Opt Express. (2016) 24:996674. doi: 10.1364/OE.24.009966

33. Lyu Y, Zou X, Shi H, Liu C, Wei C, Li J, et al. Multipulse dynamics under dissipative soliton resonance conditions. Opt Express. (2017) 25:13286-95. doi: 10.1364/OE.25.013286

34. Lyu Y, Shi H, Wei C, Li H, Li J, Liu Y. Harmonic dissipative soliton resonance pulses in a fiber ring laser at different values of anomalous dispersion. Photon Res. (2017) 5:612-6. doi: 10.1364/PRJ.5.000612

35. Chowdhury SD, Pal A, Chatterjee S, Sen R, Pal M. Multipulse dynamics of dissipative soliton resonance in an all-normal dispersion mode mocked fiber laser. J Light Technol. (2018) 36:5773-9. doi: 10.1109/JLT.2018.2877924

36. Wang Y, Zhao J, Wang S, Shu C, Su L, Tang D, et al. Unusual evolutions of dissipative-soliton-resonance pulses in an all-normal dispersion fiber laser. IEEE Photonics J. (2019) 11:1500509. doi: 10.1109/JPHOT.2019.2895687

37. Tang DY, Zhao LM, Zhao B, Liu AQ. Mechanism of multisoliton formation and soliton energy quantization in passively mode-locked fiber lasers. Phys Rev A. (2005) 72:043816. doi: 10.1103/PhysRevA.72.043816

38. Liu X. Interaction and motion of solitons in passively-mode-locked fiber lasers. Phys Rev A. (2011) 84:053828. doi: 10.1103/PhysRevA.84.053828

39. Woodward RI, Kelleher EJR. Dark solitons in laser radiation buildup dynamics. Phys Rev E. (2016) 93:032221. doi: 10.1103/PhysRevE.93. 032221

Conflict of Interest: The authors declare that the research was conducted in the absence of any commercial or financial relationships that could be construed as a potential conflict of interest.

Copyright (C) $2019 \mathrm{Lyu}$, Wei, Du, Li and Liu. This is an open-access article distributed under the terms of the Creative Commons Attribution License (CC BY). The use, distribution or reproduction in other forums is permitted, provided the original author(s) and the copyright owner(s) are credited and that the original publication in this journal is cited, in accordance with accepted academic practice. No use, distribution or reproduction is permitted which does not comply with these terms. 